\title{
Application of Differential Transformation Method to Boundary Value Problems of Order Seven and Eight
}

\author{
R. B. Ogunrinde, 0. M. Ojo \\ Department of Mathematical Sciences, Ekiti State University, Ado Ekiti, Nigeria \\ Email: rowbose@yahoo.com
}

How to cite this paper: Ogunrinde, R.B. and Ojo, O.M. (2018) Application of Differential Transformation Method to Boundary Value Problems of Order Seven and Eight. American Journal of Computational Mathematics, 8, 269-278.

https://doi.org/10.4236/ajcm.2018.83022

Received: October 5, 2017

Accepted: September 26, 2018

Published: September 29, 2018

Copyright ( 92018 by authors and Scientific Research Publishing Inc.

This work is licensed under the Creative

Commons Attribution International

License (CC BY 4.0).

http://creativecommons.org/licenses/by/4.0/

\begin{abstract}
This paper presents the use of differential transformation method (DTM), an approximating technique for solving linear higher order boundary value problems. Using DTM, approximate solutions of order seven and eight boundary value problems were developed. Approximate results are given for some examples to illustrate the efficiency and accuracy of the method. The results from this method are compared with the exact solutions.
\end{abstract}

\section{Keywords}

DTM, Differential Equation, Boundary Value Problems, Numerical Methods

\section{Introduction}

Higher order boundary value problems arise in the study of hydrodynamics and hydro magnetic stability, astronomy, fluid dynamics, astrophysics, engineering and applied physics. The boundary value problems of higher order have been investigated due to their mathematical importance and the potential for applications in diversified applied sciences [1] [2] [3].

Explicit weighting coefficients are formulated to implement the Generalized Differential Quadrature Rule (GDQR) for eighth-order differential equations. [4] [5] used Nonic spline and Non polynomial spline technique for the numerical solution of eighth-order linear special case boundary value problems. The methods presented in [6] have also been proven to be second order convergent. [7] employed finite-difference method to find the solution of eighth-order boundary value problems. [8] [9] presented an efficient numerical algorithm using Adomian decomposition method for the solutions of special eighth-order 
boundary value problem. [10] [11] [12] proposed a relatively new analytical technique, the variational iteration decomposition method (VIDM), for solving the eighth-order boundary value problems. [13] [14] [15] presented the solution of eighth order boundary value problem using octic spline. [16] presented the solutions of eighth order boundary value problems using Adomian decomposition method.

A great deal of interest has been focused on the applications of differential transformation method (DTM) to solve various scientific models [13]. In this paper, we are interested in the application of differential transformation method to solve higher order boundary value problems of order seven and eight. The concept of differential transformation method was first introduced by Zhou in 1986, and it was applied to solve linear and non-linear initial value problems in electric circuit analysis. The method can be used to evaluate the approximating solution by the finite Taylor series and by the iteration procedure describes by the transformed equations obtained from the original equation using the operations of differential transformation [11] [12].

\section{The Differential Transformation Method (DTM)}

A kth order differential transformation of a function $y(x)=f(x)$ is defined about a point $x=x_{0}$ as:

$$
Y(K)=\left[\frac{\mathrm{d}^{k} y(x)}{\mathrm{d} x^{k}}\right]_{x=x_{0}}
$$

where $k$ belongs to the set of non-negative integers, denoted as the $K$-domain.

The function $y(x)$ may be expressed in terms of the differential transforms $y(K)$ as:

$$
y(x)=\sum_{k=0}^{\infty}\left[\frac{\left(x-x_{0}\right)^{k}}{k !}\right] Y(k)
$$

Upon combining 2.1 and 2.2, we obtain: $y(x)=\sum_{k=0}^{\infty} \frac{1}{k !}\left(x-x_{0}\right)^{k}\left[\frac{\mathrm{d}^{k} y(x)}{\mathrm{d} x^{k}}\right]_{x=x_{0}}$

Which is actually the Taylor's series for $y(x)$ at about $=x_{0}$.

From the basic definition of the differential transformation, one can obtain certain laws of transformational operations, some of these, are listed in the following:

1) If $z(x)=u(x) \pm v(x)$, then $Z(k)=U(k) \pm V(k)$

2) If $z(x)=\alpha u(x)$, then $Z(k)=\alpha U(k)$

3) If $z(x)=\frac{\mathrm{d} u(x)}{\mathrm{d} x}$ then, $Z(k)=(k+1) U(k+1)$

4) If $z(x)=\frac{\mathrm{d}^{2} y(x)}{\mathrm{d} x^{2}}$ then, $Z(k)=(k+1)(k+2) U(k+2)$

5) If $z(x)=\frac{\mathrm{d}^{m} y(x)}{\mathrm{d} x^{m}}$ then, $Z(k)=(k+1)(k+2) \cdots(k+m) U(k+m)$ 
6) If $z(x)=u(x) v(x)$ then $Z(k)=\sum_{l=0}^{k} V(l) U(k-l)$

7) If $z(x)=x^{m}$ then, $Z(k)=\partial(k-n)$ where, $\partial(k-n)= \begin{cases}1 & k=n \\ 0 & k \neq n\end{cases}$

8) If $z(x)=\mathrm{e}^{\lambda x}$ then, $Z(k)=\frac{\lambda}{k !}$

9) If $z(x)=\sin (\omega x+\alpha)$ then, $Z(k)=\frac{\omega}{k !} \sin \left(\frac{\pi k}{2}+\alpha\right)$

10) If $z(x)=\cos (\omega x+\alpha)$ then, $Z(k)=\frac{\omega}{k !} \cos \left(\frac{\pi k}{2}+\alpha\right)$

\section{The Higher Order Boundary Value Problem}

1) even order boundary value problems

Consider the special $(2 \mathrm{~m})$ order BVP of the form

$$
y^{(2 m)}(x)=f(x, y), 0<x<b
$$

With boundary conditions

$$
\begin{aligned}
& y^{(2 m)}(0)=\alpha_{2 j}, j=0,1,2, \cdots,(m-1) \\
& y^{(2 m)}(b)=\beta_{2 j}, j=0,1,2, \cdots,(m-1)
\end{aligned}
$$

2) odd order boundary value problems

Consider the special $(2 m+1)$ order BVP of the form

$$
y^{(2 m+1)}(x)=f(x, y), 0<x<b
$$

With boundary conditions

$$
\begin{aligned}
& y^{(2 j+1)}(0)=\Upsilon_{2 j+1}, j=0,1,2, \cdots,(m) \\
& y^{(2 j+1)}(b)=\Upsilon_{2 j+1}, j=0,1,2, \cdots,(m)
\end{aligned}
$$

It is interesting to point out that $y(x)$ and $f(x, y)$ are assumed real and as many times differentiable as required for $x \in[0, b]$

\section{Analysis of Higher Order Boundary Value Problems by Differential Transformation}

Let the differential transform of the deflection function $y(x)$ be defined from Equation (2.1) as:

$$
Y(K)=\frac{1}{k !}\left[\frac{\mathrm{d}^{k} y(x)}{\mathrm{d} x^{k}}\right]_{x=x_{0}}
$$

where $x_{0}=0$. Also the deflection function may be expressed in terms of $Y(K)$ from Equation (2.2) as:

$$
y(x)=\sum_{k=0}^{\infty}\left[\frac{\left(x-x_{0}\right)^{k}}{k !}\right] Y(k)
$$

Now, using the transformation operations which has been formed in sec.2, 
one can obtain by taking the differential transform of Equations (3.1) and (3.4) respectively and some simplification, the following recurrence equations as $m=0,1,2, \cdots$

$$
\begin{gathered}
Y(2 m+k)=\sum_{k=0}^{\infty}\left[\frac{(2 m) !}{(2 m+k) !}\right] Y(., .) \\
Y(2 m+k+1)=\sum_{k=0}^{\infty}\left[\frac{(2 m+1) !}{(2 m+k+1) !}\right] Y(., .)
\end{gathered}
$$

where $Y(.,$.$) denotes the transformed function of linear and non linear func-$ tion $f(x, y)$. It may be noted that Equation (4.2) is independent of the boundary conditions. The differential transforms of the boundary conditions at $x=0$ are obtained from Equations (3.2) and (3.5) in the cases even order (odd order) boundary value problems respectively with the definition 4.1 as:

$$
\begin{aligned}
& Y(2 j)=\frac{1}{2 j} \alpha_{2 j}, j=0,1,2, \cdots,(2 m-1) \\
& Y(2 j+1)=\frac{1}{2 j+1} \gamma_{2 j}, j=0,1,2, \cdots, 2 m
\end{aligned}
$$

Substituting from 4.5 and 4.6 into 4.3 and 4.4 and using 4.2 , yields for $j=0,1,2, \cdots,(m-1)$

$$
y(x)=\sum_{k=0}^{\infty}\left[\frac{1}{(2 j) !} \alpha_{2 j}\right] Y(k) x^{k}
$$

And for $j=0,1,2, \cdots, m$

$$
y(x)=\sum_{k=0}^{\infty}\left[\frac{1}{(2 j+1) !} \gamma_{2 j+1}\right] Y(k) x^{k}
$$

Noting that $y^{(2 r+1)}(0)=A_{r}, \quad r=0,1,2, \cdots,(m-1)$, and $y^{(2 r)}(0)=B_{r}$, $r=0,1,2, \cdots, m$, are constants that will be approximated at the end point $x=b$.

\section{NUMERICAL EXAMPLES}

Example 1:

$$
y^{8}(x)+x y(x)=-\left(48+15 x+x^{3}\right) \mathrm{e}^{x}, 0<x<1
$$

with boundary conditions

$$
\begin{aligned}
& y(0)=0, \quad y(1)=0 \\
& y^{1}(0)=1, \quad y^{1}(1)=-\mathrm{e} \\
& y^{2}(0)=0, \quad y^{2}(1)=-4 \mathrm{e} \\
& y^{3}(0)=-3, \quad y^{3}(1)=-9 \mathrm{e}
\end{aligned}
$$

whose analytical solution is $y(x)=x(1-x) \mathrm{e}^{x}$

transforming using DTM

$$
\begin{aligned}
& Y(k+8) \\
& =\frac{k !}{(k+8) !}\left[-\frac{48}{k !}+15 \sum_{l=0}^{k} \frac{\partial(l-1)}{(k-l) !}-\sum_{l=0}^{k} \frac{\partial(l-3)}{(k-l) !}-\sum_{l=0}^{k} \partial(l-1) U(k-l)\right]
\end{aligned}
$$


With boundary conditions

$$
\begin{aligned}
& Y(0)=0, Y(1)=1, Y(2)=0, Y(3)=-\frac{3}{3 !}, \\
& Y(4)=A, Y(5)=B, Y(6)=C, Y(7)=D
\end{aligned}
$$

At $k=0$

$$
\begin{aligned}
Y(8) & =\frac{0 !}{(8) !}\left[-\frac{48}{0 !}+15 \sum_{l=0}^{0} \frac{\partial(0-1)}{(0) !}-\sum_{l=0}^{0} \frac{\partial(0-3)}{(0) !}-\sum_{l=0}^{0} \partial(0-1) U(0)\right] \\
& =\frac{-1}{840}
\end{aligned}
$$

$k=1$

$$
\begin{aligned}
Y(9) & =\frac{1 !}{(9) !}\left[-\frac{48}{1 !}+15 \sum_{l=0}^{1} \frac{\partial(1-1)}{(1) !}-\sum_{l=0}^{1} \frac{\partial(l-3)}{(1-l) !}-\sum_{l=0}^{1} \partial(l-1) Y(1-l)\right] \\
& =\frac{-1}{5760}
\end{aligned}
$$

$k=2$

$$
\begin{aligned}
Y(10) & =\frac{2 !}{(10) !}\left[-\frac{48}{2 !}+15 \sum_{l=0}^{2} \frac{\partial(1-1)}{(2-l) !}-\sum_{l=0}^{2} \frac{\partial(l-3)}{(2-l) !}-\sum_{l=0}^{2} \partial(l-1) Y(2-l)\right] \\
& =\frac{-7}{10 !}
\end{aligned}
$$

$k=3$

$$
\begin{aligned}
Y(11) & =\frac{3 !}{(11) !}\left[-\frac{48}{3 !}+15 \sum_{l=0}^{3} \frac{\partial(1-1)}{(3-l) !}-\sum_{l=0}^{3} \frac{\partial(l-3)}{(3-l) !}-\sum_{l=0}^{3} \partial(l-1) Y(3-l)\right] \\
& =\frac{-69}{11 !}
\end{aligned}
$$

$k=4$

$$
\begin{aligned}
Y(12) & =\frac{4 !}{(12) !}\left[-\frac{48}{4 !}+15 \sum_{l=0}^{4} \frac{\partial(l-1)}{(4-l) !}-\sum_{l=0}^{4} \frac{\partial(l-3)}{(4-l) !}-\sum_{l=0}^{4} \partial(l-1) Y(4-l)\right] \\
& =\frac{-1}{5702400}
\end{aligned}
$$$$
k=5
$$

$$
\begin{aligned}
Y(13) & =\frac{5 !}{(13) !}\left[-\frac{48}{5 !}+15 \sum_{l=0}^{5} \frac{\partial(l-1)}{(5-l) !}-\sum_{l=0}^{5} \frac{\partial(l-3)}{(5-l) !}-\sum_{l=0}^{5} \partial(l-1) Y(5-l)\right] \\
& =\frac{-183-A}{13 !}
\end{aligned}
$$

$k=6$

$$
\begin{aligned}
Y(14) & =\frac{6 !}{(14) !}\left[-\frac{48}{6 !}+15 \sum_{l=0}^{6} \frac{\partial(l-1)}{(6-l) !}-\sum_{l=0}^{6} \frac{\partial(l-3)}{(6-l) !}-\sum_{l=0}^{6} \partial(l-1) Y(6-l)\right] \\
& =\frac{-258-B}{14 !}
\end{aligned}
$$




$$
\begin{aligned}
& k=7 \\
& Y(15)=\frac{7 !}{(15) !}\left[-\frac{48}{7 !}+15 \sum_{l=0}^{7} \frac{\partial(l-1)}{(7-l) !}-\sum_{l=0}^{7} \frac{\partial(l-3)}{(7-l) !}-\sum_{l=0}^{7} \partial(l-1) Y(7-l)\right] \\
& =\frac{-363-C}{15 !} \\
& k=8 \\
& Y(16)=\frac{8 !}{(16) !}\left[-\frac{48}{8 !}+15 \sum_{l=0}^{8} \frac{\partial(l-1)}{(8-l) !}-\sum_{l=0}^{8} \frac{\partial(l-3)}{(8-l) !}-\sum_{l=0}^{8} \partial(l-1) Y(8-l)\right] \\
& =\frac{-504-D}{16 !} \\
& y(x)=\sum_{k=0}^{n} Y(k) x^{k} \\
& y(x)=x-\frac{3}{3 !} x^{3}+A x^{4}+B x^{5}+C x^{6}+D x^{7}-\frac{1}{840} x^{8}-\frac{1}{5760} x^{9} \\
& -\frac{79}{10 !} x^{10}-\frac{69}{11 !} x^{11}-\frac{1}{5702400} x^{12}-\frac{183+A}{13 !} x^{13} \\
& -\frac{258+B}{14 !} x^{14}-\frac{363+C}{15 !} x^{15}-\frac{504+D}{16 !} x^{16}
\end{aligned}
$$

Using the boundary conditions given in (2), the required equation is (Table 1)

$$
\begin{aligned}
Y(x)= & x-\frac{3}{3 !} x^{3}-0.180317402 x^{4}-0.492347647 x^{5}+0.272873875 x^{6} \\
& -0.094455951 x^{7}-\frac{1}{840} x^{8}-\frac{1}{5760} x^{9}-\frac{79}{10 !} x^{10}-\frac{69}{11 !} x^{11} \\
& -\frac{1}{5702400} x^{12}-\frac{181.8196826}{13 !} x^{13}-\frac{257.5076524}{14 !} x^{14} \\
& -\frac{363.2728476}{15 !} x^{15}-\frac{503.905544}{16 !} x^{16}
\end{aligned}
$$

Example 2:

Consider a $7^{\text {th }}$ order linear boundary value problem

Table 1. Results of Problem 1 for $h=0.1$.

\begin{tabular}{cccc}
\hline$X$ & Analytical solution $(h=0.1)$ & $D T M(h=0.1)$ & Error \\
\hline 0.0 & 0.0000 & 0.0000 & 0.0000 \\
0.1 & 0.099465382 & 0.099477308 & $1.1926 \times 10^{-5}$ \\
0.2 & 0.195424441 & 0.19557019 & $1.45749 \times 10^{-4}$ \\
0.3 & 0.283470349 & 0.284021212 & $5.50863 \times 10^{-4}$ \\
0.4 & 0.358037927 & 0.359304348 & $1.266421 \times 10^{-3}$ \\
0.5 & 0.412180317 & 0.414365019 & $2.184702 \times 10^{-3}$ \\
0.6 & 0.437308512 & 0.440411098 & 0.028230781 \\
0.7 & 0.422888068 & 0.426705169 & $3.817101 \times 10^{-3}$ \\
0.8 & 0.356086548 & 0.360307456 & $4.220908 \times 10^{-3}$ \\
0.9 & 0.22136428 & 0.225683179 & $4.318899 \times 10^{-3}$ \\
1.0 & 0 & $4.365206367 \times 10^{-3}$ & $4.365206367 \times 10^{-3}$ \\
\hline
\end{tabular}




$$
U^{7}(x)=x u(x)+\mathrm{e}^{x}\left(x^{2}-2 x-6\right), 0 \leq x \leq 1
$$

Subject to the boundary conditions

$$
\begin{aligned}
& U(0)=1, U(1)=0 \\
& U^{1}(0)=0, U^{1}(1)=-\mathrm{e} \\
& U^{2}(0)=-1, U^{2}(1)=-2 \mathrm{e} \\
& U^{3}(0)=-2
\end{aligned}
$$

Whose analytical solution is $U(x)=(1-x) \mathrm{e}^{x}$

\section{Transformed formular is}

$$
\begin{aligned}
& U(k+7) \\
& =\frac{k !}{(k+7) !}\left[\sum_{l=0}^{k} \partial(l-1) U(k-l)+\sum_{l=0}^{k} \frac{\partial(l-2)}{(k-l) !}-2 \sum_{l=0}^{k} \frac{\partial(l-1)}{(k-l) !}-\frac{6}{k !}\right]
\end{aligned}
$$

Transformed boundary conditions are

$$
\begin{aligned}
& U(0)=1, U(1)=0, U(2)=\frac{-1}{2 !}, U(3)=\frac{-2}{3 !}, \\
& U(4)=P, U(5)=Q, U(6)=R
\end{aligned}
$$

at $k=1$

$$
\begin{aligned}
U(8) & =\frac{1 !}{(8) !}\left[\sum_{l=0}^{1} \partial(l-1) U(1-l)+\sum_{l=0}^{1} \frac{\partial(l-2)}{(1-l) !}-2 \sum_{l=0}^{1} \frac{\partial(l-1)}{(1-l) !}-\frac{6}{1 !}\right] \\
& =\frac{-1}{5760}
\end{aligned}
$$

$k=2$

$$
\begin{aligned}
U(9) & =\frac{2 !}{(9) !}\left[\sum_{l=0}^{2} \partial(l-1) U(2-l)+\sum_{l=0}^{2} \frac{\partial(l-2)}{(2-l) !}-2 \sum_{l=0}^{2} \frac{\partial(l-1)}{(2-l) !}-\frac{6}{2 !}\right] \\
& =\frac{-1}{45360}
\end{aligned}
$$

$k=3$

$$
\begin{aligned}
U(10) & =\frac{3 !}{(10) !}\left[\sum_{l=0}^{3} \partial(l-1) U(3-l)+\sum_{l=0}^{3} \frac{\partial(l-2)}{(3-l) !}-2 \sum_{l=0}^{3} \frac{\partial(l-1)}{(3-l) !}-\frac{6}{3 !}\right] \\
& =\frac{-1}{403200}
\end{aligned}
$$

$k=4$

$$
\begin{aligned}
U(11) & =\frac{4 !}{(11) !}\left[\sum_{l=0}^{4} \partial(l-1) U(4-l)+\sum_{l=0}^{4} \frac{\partial(l-2)}{(4-l) !}-2 \sum_{l=0}^{4} \frac{\partial(l-1)}{(4-l) !}-\frac{6}{4 !}\right] \\
& =\frac{-1}{3991680}
\end{aligned}
$$

$k=5$

$$
\begin{aligned}
U(12) & =\frac{5 !}{(12) !}\left[\sum_{l=0}^{5} \partial(l-1) U(5-l)+\sum_{l=0}^{5} \frac{\partial(l-2)}{(5-l) !}-2 \sum_{l=0}^{5} \frac{\partial(l-1)}{(5-l) !}-\frac{6}{5 !}\right] \\
& =\frac{1+30 P}{119250400}
\end{aligned}
$$




$$
\begin{aligned}
& k=6 \\
& U(13)=\frac{6 !}{(13) !}\left[\sum_{l=0}^{6} \partial(l-1) U(6-l)+\sum_{l=0}^{6} \frac{\partial(l-2)}{(6-l) !}-2 \sum_{l=0}^{6} \frac{\partial(l-1)}{(6-l) !}-\frac{6}{6 !}\right] \\
& =\frac{1+60 Q}{518918400} \\
& k=7 \\
& U(14)=\frac{7 !}{(14) !}\left[\sum_{l=0}^{7} \partial(l-1) U(7-l)+\sum_{l=0}^{7} \frac{\partial(l-2)}{(7-l) !}-2 \sum_{l=0}^{7} \frac{\partial(l-1)}{(7-l) !}-\frac{6}{7 !}\right] \\
& =\frac{22+7 ! R}{14 !} \\
& U(x)=\sum_{k=0}^{n} U(k) x^{k} \\
& =1-\frac{1}{2} x^{2}-\frac{1}{3} x^{3}+P x^{4}+Q x^{5}+R x^{6}-\frac{1}{840} x^{7}-\frac{1}{5760} x^{8} \\
& -\frac{1}{45360} x^{9}-\frac{1}{403200} x^{10}-\frac{1}{3991680} x^{11} \\
& +\frac{1+30 P}{119750680} x^{12}+\frac{1+60 Q}{518918400} x^{13}+\frac{22+7 ! R}{14 !} x^{14}
\end{aligned}
$$

Using the conditions given in (2), the required equation is (Table 2)

$$
\begin{aligned}
U(x)= & 1-\frac{1}{2} x^{2}-\frac{1}{3} x^{3}-0.125004443 x^{4}-0.033324638 x^{5} \\
& -6.948728418 \times 10^{-3} x^{6}-\frac{1}{840} x^{7}-\frac{1}{5760} x^{8}-\frac{1}{45360} x^{9} \\
& -\frac{1}{403200} x^{10}-\frac{1}{3991680} x^{11}-\frac{2.75013329}{119750400} x^{12} \\
& -\frac{0.99947828}{518918400} x^{13}-\frac{13.02159123}{14 !} x^{14}
\end{aligned}
$$

Table 2. Results of Problem 2 for $h=0.1$.

\begin{tabular}{cccc}
\hline$X$ & Analytical solution $(h=0.1)$ & $D T M(h=0.1)$ & Error \\
\hline 0.0 & 1 & 1 & 0 \\
0.1 & 0.994653826 & 0.994653825 & $1 \times 10^{-9}$ \\
0.2 & 0.977122206 & 0.977122201 & $5 \times 10^{-9}$ \\
0.3 & 0.944901165 & 0.944901147 & $1.8 \times 10^{-8}$ \\
0.4 & 0.895094878 & 0.895094776 & $4.2 \times 10^{-8}$ \\
0.5 & 0.824360635 & 0.824360563 & $7.2 \times 10^{-8}$ \\
0.6 & 0.72884752 & 0.728847377 & $1.43 \times 10^{-7}$ \\
0.7 & 0.604125812 & 0.604125704 & $1.08 \times 10^{-7}$ \\
0.8 & 0.445108185 & 0.445108094 & $9.1 \times 10^{-8}$ \\
0.9 & 0.245960311 & 0.245960253 & $5.8 \times 10^{-8}$ \\
1.0 & 0 & $5.621870471 \times 10^{-5}$ & $5.6 \times 10^{-5}$ \\
\hline
\end{tabular}




\section{Conclusion}

In this paper, the differential transformation method is used to find the solution of higher order boundary value problems (order seven and eight). The results show that the convergence and accuracy of the method for numerically analysed eight order boundary value problem are in agreement with the analytical solutions. The method is easy to apply and can be applied easily to similar problems that engineering problems. Further work can be done on higher orders.

\section{Conflicts of Interest}

The authors declare no conflicts of interest regarding the publication of this paper.

\section{References}

[1] Akram, G. and Rehman H.U. (2011) Solution of First Order Singularly Perturbed Initial Value Problem in Reproducing Kernel Hilbert Space. European Journal of Scientific Research, 53, 516-523.

[2] Chandrasekhar, S. (1961) Hydrodynamic and Hydromagnetic Stability. Oxford, Clarendon Press.

[3] Siddiqi, S.S. and Akram, G. (2007) Solution of Eighth-Order Boundary Value Problems Using the Nonpolynomial Spline Technique. International Journal of Computer Mathematics, 84, 347-368. https://doi.org/10.1080/00207160601177226

[4] Bishop, R.E.D., Cannon, S.M. and Miao S. (1989) On Coupled Bending and Torsional Vibration of Uniform Beams. Journal of Sound and Vibration, 131, 457-464. https://doi.org/10.1016/0022-460X(89)91005-5

[5] Liu, G.R. and Wu, T.Y. (2002) Differential Quadrature Solutions of Eighth-Order Boundary-Value Differential Equations. Journal of Computational and Applied Mathematics, 145, 223-235. https://doi.org/10.1016/S0377-0427(01)00577-5

[6] He, J.-H. (2007) The Variational Iteration Method for Eighth-Order Initial-Boundary Value Problems. Physica Scripta, 76, 680-682. https://doi.org/10.1088/0031-8949/76/6/016

[7] Akram, G. and Siddiqi, S.S. (2006) Nonic Spline Solutions of Eighth Order Boundary Value Problems. Applied Mathematics and Computation, 182, 829-845. https://doi.org/10.1016/j.amc.2006.04.046

[8] Li, C. and Cui, M. (2003) The Exact Solution for Solving a Class of Nonlinear Operator Equations in the Reproducing Kernel Space. Applied Mathematics and Computation, 143, 393-399. https://doi.org/10.1016/S0096-3003(02)00370-3

[9] Ghazala, A. and Hamood, U.R. (2013) Numerical Solution of Eight Order Boundary Value Problems in Reproducing Kernel Space. Numerical Algorithm, 62, 527-540. https://doi.org/10.1007/s11075-012-9608-4

[10] Golbabai, A. and Javidi, M. (2007) Application of Homotopy Perturbation Method for Solving Eighth Order Boundary Value Problems. Applied Mathematics and Computation, 191, 334-346. https://doi.org/10.1016/j.amc.2007.02.091

[11] Porshokouhi, M.G., Ghanbari, B., Gholami, M. and Rashidi, M. (2011) Numerical Solution of Eighth Order Boundary Value Problems with Variational Iteration Method. General Mathematics Notes, 2, 128-133.

[12] Boutayeb, A. and Twizell, E.H. (1993) Finite-Difference Methods for the Solution of 
Eighth-Order Boundary-Value Problems. International Journal of Computer Mathematics, 48, 63-75. https://doi.org/10.1080/00207169308804193

[13] Inc, M. and Evans, D.J. (2004) An Efficient Approach to Approximate Solutions of Eighth-Order Boundary Value Problems. International Journal of Computer Mathematics, 81, 685-692. https://doi.org/10.1080/0020716031000120809

[14] Wazwaz, A.M. (2000) The Numerical Solutions of Special Eighth-Order Boundary Value Problems by the Modified Decomposition Method. Neural, Parallel, and Scientific Computations, 8, 133-146.

[15] Mestrovic, M. (2007) The Modified Decomposition Method for Eighth-Order Boundary Value Problems. Applied Mathematics and Computation, 188, 1437-1444. https://doi.org/10.1016/j.amc.2006.11.015

[16] Noor, M.A. and Mohyud-Din, S.T. (2007) Variational Iteration Decomposition Method for Solving Eighth-Order Boundary Value Problems. Differential Equations and Nonlinear Mechanics, 2007, 16. https://doi.org/10.1155/2007/19529 